\title{
Virtual Network Embedding Based on Complex Network Theory
}

\author{
Junwei Ge $e^{1, a}$, Ruizhi Yuan ${ }^{2, b}$ and Yiqiu Fang ${ }^{3, c}$ \\ ${ }^{1,3}$ School of Computer Science and Technology, Chongqing University of Posts and \\ Telecommunications, Chongqing 400065, China \\ ${ }^{2}$ School of Communication and Information Engineering, Chongqing University of Posts and \\ Telecommunications, Chongqing 400065, China \\ agejw@cqupt.edu.cn, ${ }^{b} 13618264745 @ 163 . c o m,{ }^{c}$ fangyq@cqupt.edu.cn
}

Keywords: virtual network, betweenness centrality based on random walks, cluster coefficient.

\begin{abstract}
How to effectively map the virtual network in the network infrastructure is a huge challenge in network virtualization. In this paper, for the first time, we evaluate the global network node weight according to the betweenness centrality based on random walks, then introduce cluster coefficient theory into the virtual network embedding to evaluate the topological information and bandwidth resource of the link local which is around the target node to complete the twice evaluation of node. Based on the two points above, we put forward the virtual network mapping algorithm based on social network centrality. Experimental results show that the new algorithm can increase the revenue/cost $(\mathrm{R} / \mathrm{C})$ ratio and acceptance ratio.
\end{abstract}

\section{Introduction}

The Internet has become an important information infrastructure to support the modern social economic development and technological innovation. However, the Internet service model which only provides "best effort" services is faced with serious technical challenges in security, mobility and quality of service ${ }^{[1]}$. To solve problems above, the researchers have put forward the network virtualization as one way to get rid of the future network gridlock.

A fundamental problem in network virtualization is the virtual network embedding problem. At this stage many measures of virtual network embedding by exploiting topological information have been put forward, but most of them just take the node weight of global network into account and ignore the local link topology information and resource property which is around the target node.

To solve the problems above, we evaluate the global network node weight according to the betweenness centrality based on random walks, then introduce and improve cluster coefficient theory into the virtual network embedding to evaluate the local link topological information and resource property which is around the target node to complete the twice evaluation of node. Based on the two points above, we put forward the virtual network mapping algorithm based on social network centrality.

\section{Virtual Network Embedding Problem}

\subsection{Network Model}

The substrate network is modeled as a weighted graph $\mathrm{G}_{\mathrm{s}}=\left(N_{s}, L_{s}, A_{s}^{n}, A_{s}^{l}\right)$. The virtual networks are defined similarly as $\mathrm{G}_{\mathrm{v}}=\left(N_{\mathrm{v}}, L_{\mathrm{v}}, \mathrm{C}_{\mathrm{v}}^{n}, C_{\mathrm{v}}^{l}\right)$. Virtual network mapping problem can be defined as: $\mathrm{M}: G_{v}\left(N_{v}, L_{v}\right) \rightarrow G_{s}\left(N_{s}^{\prime}, P_{s}^{\prime}\right)$.The virtual network mapping processes are broken up into two steps: 1)mapping the virtual nodes to the substrate nodes meeting the resource constraints; 2)mapping the virtual links to substrate links meeting the bandwidth resource constraints.

\subsection{Objectives of Virtual Network Embedding}

One important objective of VN embedding is acceptance ratio, which is to achieve a high acceptance ratio of the virtual network requests over time. It can be defined as: 


$$
\lim _{T \rightarrow \infty} \frac{\sum_{t=0}^{T} \mathrm{~V}_{\mathrm{s}}}{\sum_{t=0}^{T} V}
$$

Another important objective of $\mathrm{VN}$ embedding is the long-term revenue to cast ratio, which denotes the economic benefits of accepting VN requests and the cost of an InP. It can be defined as:

$$
\lim _{T \rightarrow \infty} \frac{\sum_{t=0}^{T} R\left(G_{v}, t\right)}{\sum_{t=0}^{T} \mathrm{C}\left(\mathrm{G}_{\mathrm{v}}, \mathrm{t}\right)}
$$

\section{3 the VN Mapping Algorithm Based on Social Network Centrality(RWC)}

In this paper, we evaluate the global network node weight according to the betweenness centrality based on random walks, then introduce and improve cluster coefficient theory into the virtual network embedding to evaluate the local link topology information and resource property which is around the target node, completing the twice evaluation of node. Based on the two points above, we put forward the virtual network mapping algorithm based on social network centrality(RWC).

Definition 1 The betweenness centrality based on random walks ${ }^{[2]}$ :

$$
\mathrm{C}_{\mathrm{r}}\left(n_{i}\right)=\frac{\sum_{s<t} I_{s t}^{i}}{n(n-1) / 2}
$$

The random walks betweenness reflects the importance of nodes in the network and gives support to the nodes mapping from a global perspective.

Definition 2 Cluster coefficient ${ }^{[3]}$ :

$$
\mathrm{C}_{\mathrm{i}}^{w}=\frac{1}{s_{i}\left(k_{i}-1\right)} \sum_{j, k} \frac{\left(w_{i j}+w_{i k}\right)}{2} a_{i j} a_{i k} a_{j k}
$$

Cluster coefficient reflects the connectivity of the nodes around the node $\mathrm{n}_{i}$.

Based on the Cluster coefficient theory, as well taking into account the link bandwidth and topological information, we define the Cluster coefficient as follows:

$$
\mathrm{C}_{i b}=\frac{C_{r}\left(\mathrm{n}_{i}\right)}{\sum_{j=1}^{N} a_{i j} b(i, j)\left(a_{i j}-1\right)} \sum_{j, k} \frac{(b(i, j)+b(j, k)+b(i, k))}{3} a_{i j} a_{i k} a_{j k}
$$

Where $C_{r}\left(\mathrm{n}_{i}\right)$ is the weight of node $\mathrm{n}_{i}$ computed in formula (1), $b(i, j)$ is the link bandwidth between node $n_{i}$ and $n_{j}$. In the process of virtual network mapping, the link between node $n_{i}$ and $\mathrm{n}_{k}$ is also important, $\operatorname{sob}(j, k)$ is taken into account as well.

The algorithm RWC maps the virtual nodes based on the Breadth-first Search strategy. RWC firstly computes $\mathrm{C}_{\mathrm{ib}}$ values of all virtual nodes, then construct Breadth-first Search tree based on virtual network topology. Sorting nodes of every level in non- increasing order according to their $\mathrm{C}_{\mathrm{ib}}$ values. Bigger the $\mathrm{C}_{\mathrm{ib}}$ value of a node is, higher the mapping priority is. The algorithm RWC begins mapping the virtual network from the root of Breadth-first Search tree and mapping the virtual link based on K shortest paths algorithm(KSP $)^{[4]}$. The algorithm is described as follows: 


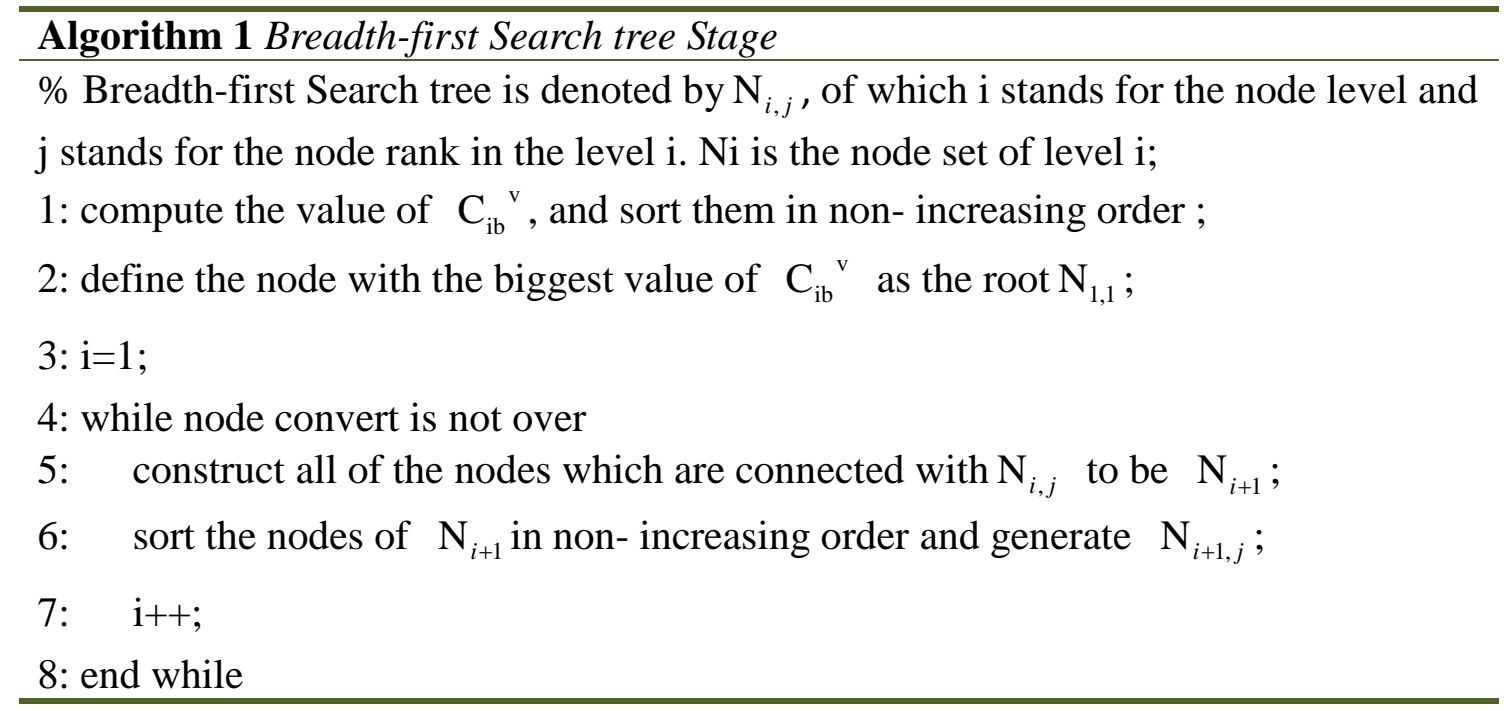

Algorithm 2 RWC Mapping Stage

1: compute the value of $\mathrm{C}_{\mathrm{ib}}{ }^{\mathrm{s}}$, and sort them in non- increasing order ;

2: mapping of Breadth-first Search tree $\mathrm{N}_{i, j}$ :

(1) the mapping begins from the root $\mathrm{N}_{1,1}$ and mapping next levels in turn;

(2) map the substrate nodes which are connected with substrate nodes $N_{i-1}$, never

have been mapped, meet the node resource requirements and with the biggest value of $\mathrm{C}_{\mathrm{ib}}{ }^{\mathrm{v}}$. If (2) fails, goto (3);

(3) map the substrate nodes which are 2 hop count from nodes $N_{i-1}$, never have been mapped, meet the node resource requirements and with the biggest value of $\mathrm{C}_{\mathrm{ib}}{ }^{\mathrm{v}}$;

3: if any node in $\mathrm{N}_{i, j}$ is not mapped, goto step 2;

4: map the virtual link based on K shortest paths algorithm;

5: update the resource of $\mathrm{G}_{s}$.

\section{Performance Evaluation}

The virtual networks and substrate network are both generated by the GT-ITM tool. The substrate network is generated with 100 nodes and about 500 links.The CPU of nodes and bandwidth of links in substrate follow a uniform distribution from 40 to 100 . The simulation includes 2500 virtual network requirements. The number of nodes in each VN request follows a uniform distribution between 2 and 10, and the CPU of nodes and bandwidth of links follow a uniform distribution from 0 to 50 . The probability of each pair of virtual nodes connection is $50 \%$. The runtime of each VN request is exponentially distributed with a mean of 1000 time units, and the VN requests arrive following a Poisson distribution with a mean of five requests in 100 time units. The runtime of simulation is 50000 time units.

The objectives of virtual network embedding are acceptance ratio and revenue to cast ratio(R/C). The algorithms compared are Greedy algorithm ${ }^{[5]}(\mathrm{BL})$ and Closeness

Centrality $^{[6]}(\mathrm{CL})$. 


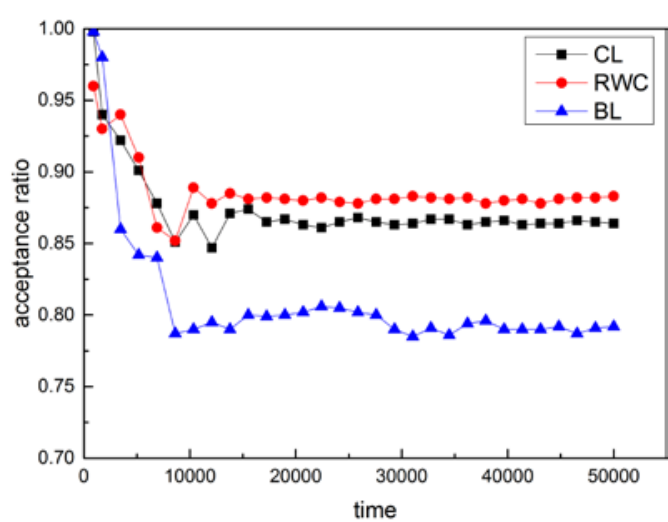

Figure 1.Acceptance ratio

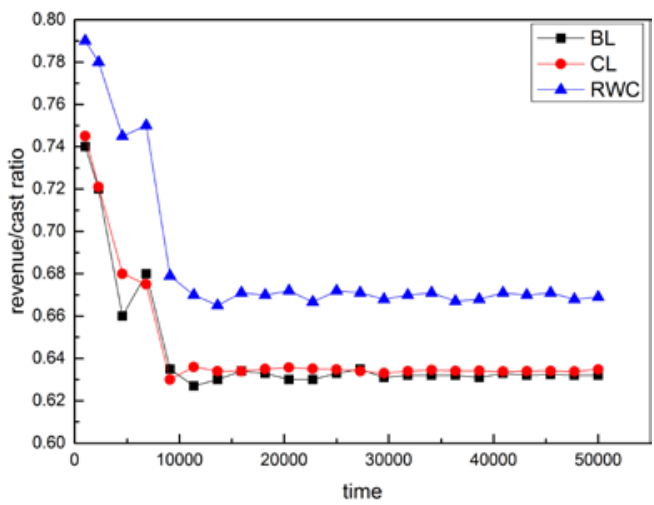

Figure 2.Revenue/cast ratio

Figure 1 shows the acceptance ratio of three algorithms. The acceptance ratio of RWC algorithm(88\%) is $11 \%$ higher than the acceptance ratio of Greedy algorithm(79\%), and 2\% higher than Closeness Centrality algorithm(86\%). The reason is that RWC algorithm not only analyses the weight of global network nodes, but also takes into account the connectivity and bandwidth resource of the link local.

Figure 2 shows the revenue/cast ratio of three algorithms. The revenue/cast ratio of Greedy and Closeness Centrality algorithm is about 63\%. The revenue/cast ratio of RWC algorithm is about $67 \%$, which is $6.3 \%$ higher than the other two algorithms. Because RWC algorithm analyses the topological information of the link. It decreases the number of substrate network link and reduces the cost of substrate network.

\section{Conclusion}

In this paper, we research the virtual network from the perspective of network topology, and put forward the virtual network mapping algorithm based on social network centrality. The algorithm introduces the betweenness centrality based on random walks theory and cluster coefficient theory, and analyzes the notwork from both global and local aspects. Experimental results show that RWC algorithm can increase the revenue/cost $(\mathrm{R} / \mathrm{C})$ ratio and acceptance ratio.

\section{References}

[1] Turner J S, Taylor D E. Diversifying the Internet[C]// Global Telecommunications Conference, 2005. GLOBECOM '05. IEEE. IEEE, 2005:6 pp.-760.

[2] Newman M E J. A measure of betweenness centrality based on random walks[J]. Social Networks, 2003, 27(1):39 - 54.

[3] Barrat, A, et al. "The architecture of complex weighted networks."Proceedings of the National Academy of Sciences 101.11(2004):3747-3752.

[4] De xin Y U, Yang Z S, Gao P. Constrained K-shortest paths algorithm within dynamic restricted searching area[J]. Journal of Jilin University, 2009.

[5] Zhu Y, Ammar M. Algorithms for Assigning Substrate Network Resources to Virtual Network Components.[J]. Proceedings - IEEE INFOCOM, 2006:1 - 12.

[6] Wang Z, Han Y, Lin T, et al. Virtual network embedding by exploiting topological information[C]// IEEE Global Communications Conference. IEEE, 2012:2603-2608. 